\title{
Embryos grown in the dead zone
}

\section{Assembling the first protoplanetary cores in low mass self-gravitating circumstellar disks of gas and solids ${ }^{\star}$}

\author{
W. Lyra ${ }^{1}$, A. Johansen ${ }^{2}$, H. Klahr ${ }^{3}$, and N. Piskunov ${ }^{1}$ \\ ${ }^{1}$ Department of Physics and Astronomy, Uppsala Astronomical Observatory, Box 515, 75120 Uppsala, Sweden \\ e-mail: wlyra@astro.uu.se \\ 2 Leiden Observatory, Leiden University, PO Box 9513, 2300 RA Leiden, The Netherlands \\ 3 Max-Planck-Institut für Astronomie, Königstuhl 17, 69117 Heidelberg, Germany
}

Received 16 July 2008 / Accepted 10 October 2008

ABSTRACT

\begin{abstract}
Context. In the borders of the dead zones of protoplanetary disks, the inflow of gas produces a local density maximum that triggers the Rossby wave instability. The vortices that form are efficient in trapping solids.

Aims. We aim to assess the possibility of gravitational collapse of the solids within the Rossby vortices.

Methods. We perform global simulations of the dynamics of gas and solids in a low mass non-magnetized self-gravitating thin protoplanetary disk with the Pencil Code. We use multiple particle species of radius 1, 10,30, and $100 \mathrm{~cm}$. The dead zone is modelled as a region of low viscosity.

Results. The Rossby vortices excited in the edges of the dead zone are efficient particle traps. Within 5 orbits after their appearance, the solids achieve critical density and undergo gravitational collapse into Mars sized objects. The velocity dispersions are of the order of $10 \mathrm{~m} \mathrm{~s}^{-1}$ for newly formed embryos, later lowering to less than $1 \mathrm{~m} \mathrm{~s}^{-1}$ by drag force cooling. After 200 orbits, over 300 gravitationally bound embryos were formed, 20 of them being more massive than Mars. Their mass spectrum follows a power law of index $-2.3 \pm 0.2$.
\end{abstract}

Key words. accretion, accretion disks - instabilites - stars: planetary systems: formation

\section{Introduction}

The formation of planets is one of the major unsolved problems in modern astrophysics. In the standard core accretion scenario, sub- $\mu \mathrm{m}$ grains assemble into progressively larger bodies through electrostatic interactions (Natta et al. 2007), eventually growing into centimeter and meter sized boulders. Growth beyond this size, however, is halted since these boulders have very poor sticking properties and are easily destroyed by collisions at the velocities assumed to be prevalent in circumstellar disks (Benz 2000). Furthermore, centimeter and meter sized solids are loosely decoupled from the gas, but remain sufficiently small to be affected by significant gas drag. The resulting headwind from the sub-Keplerian gas reduces their angular momentum and forces them into spiral trajectories onto the star in timescales as short as a few thousand years (Weidenschilling 1977a).

A mechanism for overcoming these barriers was presented by Kretke \& Lin (2007). In the presence of sufficient ionization, the gaseous disk couples with the ambient weak magnetic field, which triggers the growth of the magneto-rotational instability (MRI; Balbus \& Hawley 1991). In its saturated state, a vigorous turbulence drives accretion onto the star by means of magnetic and kinetic stresses. However, in the water condensation front (snowline) the abundant presence of snowflakes effectively removes free electrons from the gas, lowering the degree of ionization. The turbulence is weakened locally and the accretion flow

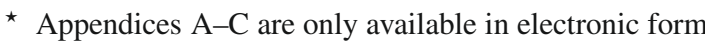
at http://www . aanda.org
}

is stalled. As the radial inflow proceeds from the outer disk, gas accumulates at the snowline. Since embedded solid bodies move towards gas pressure maxima (Haghighipour \& Boss 2003), the snowline environment proposed by Kretke \& Lin (2007) is potentially an efficient particle trap. This scenario was further explored by Brauer et al. (2008), who demonstrated that as solids concentrate at this local pressure maximum, rapid growth into kilometer sized planetesimals occurs by coagulation.

Kretke et al. (2008) emphasized that an identical mechanism is supposed to occur elsewhere in the disk. Ionization ought to be present in the very inner disk due to the high temperatures, as well as in the outer regions where the gas is sufficiently thin for cosmic rays to penetrate to the disk midplane and provide ionization throughout. In between, however, temperatures are too low and gas columns too thick to allow sufficient ionization either by collisions or by cosmic rays. In the midplane of this region, the gas is neutral and the turbulence is largely suppressed (Gammie 1996). As in the snowline, the accretion flow from the MRI-active regions halts at the borders of this "dead" zone, where the gas then accumulates.

These models have been tested only by one-dimensional simulations, and these tests have therefore not benefitted from an interesting development. As shown by Varnière \& Tagger (2006), the density pileup at the border of the dead zone triggers the Rossby wave instability (RWI; Li et al. 2001). The azimuthal symmetry of the problem is broken and long-lived anticyclonic vortices are formed as the waves break and coalesce. Such entities are of significant interest because, by rotating clockwise 


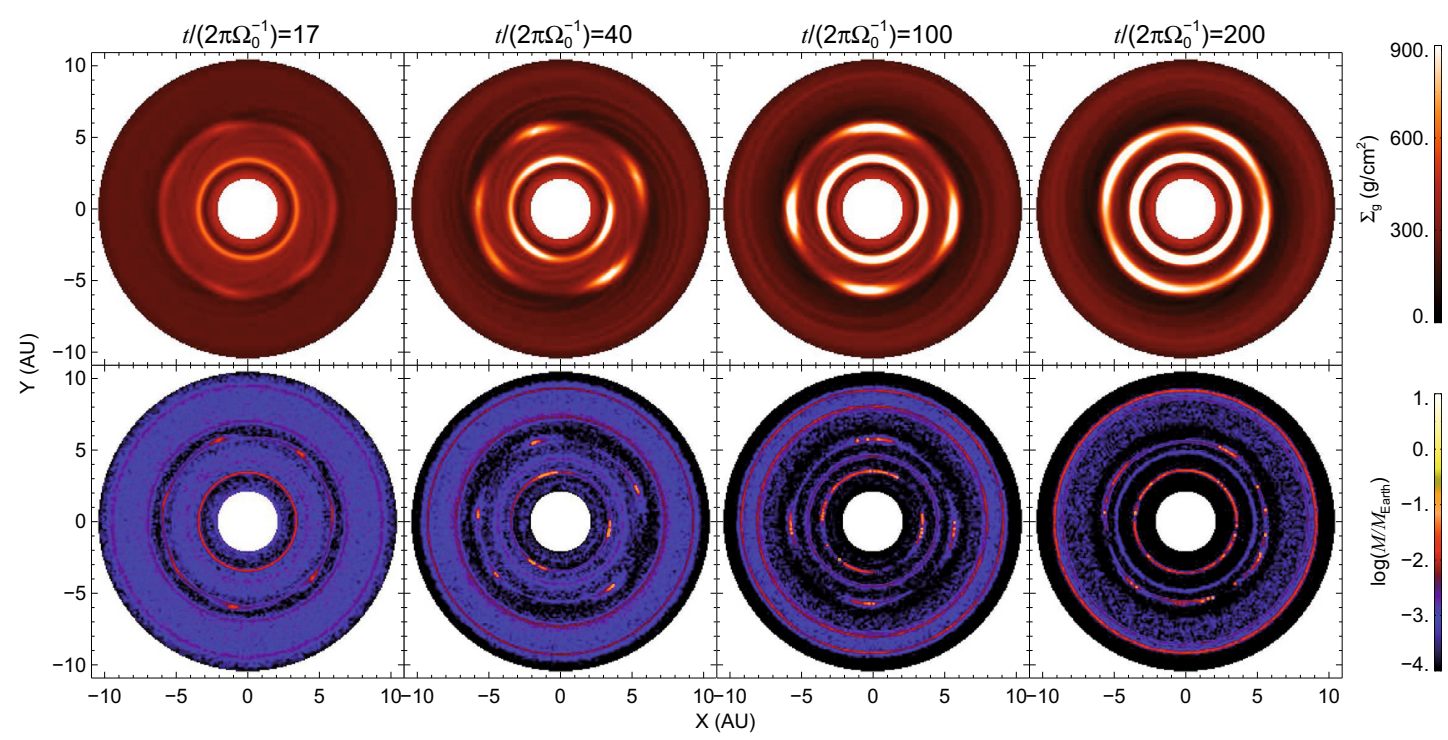

Fig. 1. The appearance of the disk in the gas (upper panels) and solid (lower panels) phases in selected snapshots. The Rossby vortices first appear at 15 orbits. Collapse of the particles into a gravitationally bound planetary embryo the size of Mars occurs 5 orbits later. The vortices tend to merge and decrease in number, retaining an $m=4$ dominant mode at the outer edge until the end of the simulation, up to which over 300 embryos were formed.

in the global counterclockwise Keplerian flow, they amplify the local shear and induce a net force on solid particles towards their center (Barge \& Sommeria 1995). As shown by Klahr \& Bodenheimer (2006), the accumulation of solids under these circumstances is likely to lead to high densities. Inaba \& Barge (2006) continued the study of Varnière \& Tagger (2006) by including solids and confirming that the Rossby vortices excited in the borders of the dead zone act as powerful traps, enhancing the local solids-to-gas ratio by at least an order of magnitude. Unfortunately, they used a fluid approach - which limited the maximum particle size they could consider - and they did not include the self-gravity of the solids, which is crucial to follow the gravitational collapse. Studies with interacting particles in the literature include a MRI-unstable local box (Johansen et al. 2007) capable of producing dwarf planets out of meter sized boulders, a global massive disk unstable to gas self-gravity (Rice et al. 2006) in which concentrations of $0.5 M_{\oplus}$ are seen in the spiral arms, and a simulation that produces $10-100 \mathrm{~km}$ "sandpile" clumps formed out of mm-sized particles (Cuzzi et al. 2008).

In this letter, we build on the studies of Varnière \& Tagger (2006) and Inaba \& Barge (2006) by including self-gravitating centimeter and meter sized Lagrangian particles to model the solid phase. We show that in the vortices launched by the RWI in the borders of the dead zone, the solids quickly achieve critical densities and undergo gravitational collapse into protoplanetary embryos in the mass range $0.1-0.6 M_{\oplus}$.

\section{Model}

We work in the thin disk approximation, using the vertically averaged equations of hydrodynamics. The gas drag is implemented in the same way as Paardekooper (2007), interpolating between Epstein and Stokes drag (see online supplement). The back reaction of the drag force onto the gas is present. The Poisson solver for the particles is a particle-mesh solver based on multiple Fourier transforms in a Cartesian grid, as used by Johansen et al. (2007).

We follow the Varnière \& Tagger (2006) dead zone model, which consists of jumps in the viscosity profile. We artificially place the inner and outer edges of the dead zone at 0.6 and 1.2 times the semi-major axis of Jupiter (5.2 AU), using Heaviside functions to jump from $\alpha=10^{-2}$ to zero inside the dead zone. The parameter $\alpha$ is the usual alpha viscosity (Shakura \& Sunyaev 1973).

The simulations were performed with the PENCIL $\mathrm{CODE}^{1}$ in Cartesian geometry. The resolution was $256 \times 256$. The surface density profile followed a power law of $\Sigma(r) \propto r^{-1 / 2}$, and we chose a disk about twice as massive as the Minimum Mass Solar Nebula, with surface density $\Sigma_{0}=300 \mathrm{~g} \mathrm{~cm}^{-2}$ at $5.2 \mathrm{AU}$. The sound speed followed the local isothermal approximation with a radial temperature profile $T(r) \propto r^{-1}$. The disk aspect ratio was $h=0.05$. For the solids, we used $10^{5}$ Lagrangian superparticles and the interstellar value for the solids-to-gas ratio $\left(10^{-2}\right)$. Each superparticle therefore contained $10^{-9} M_{\odot} \simeq 2 \times 10^{24} \mathrm{~g}$ of material. We used multiple particles species, of $1,10,30$, and $100 \mathrm{~cm}$ radii, each represented by $1 / 4$ of the total number of particles. We quote time as orbital periods at 5.2 AU.

\section{Results}

In Fig. 1, we show snapshots of the appearance of the disk for the gas and solids phases. The vortices triggered by the Rossby wave instability are visible as early as 15 orbits. As seen in the solids phase, the particles are trapped by the vortical motion and soon reach extremely high densities. After 17 orbits, seven vortices appear at the outer edge. After 45 orbits have elapsed, the $m=4$ mode begins to dominate, persisting until the end of the simulation at 200 orbits, their gas surface density peaking at 4.5 times their initial value. In the inner edge of the dead zone, at 40 orbits we see a conspicuous $m=3$ mode. By the end of the simulation, their surface density has increased by a factor of 8 relative to the initial condition, and a weak $m=2$ mode is visible, albeit with far less contrast than in the outer disk.

In Fig. 2a, we plot the time evolution of the maximum concentration of solids. The solid line represents the maximum mass of solids contained in a single cell. The red dashed line marks the

\footnotetext{
${ }^{1}$ See http://www.nordita.org/software/pencil-code
} 

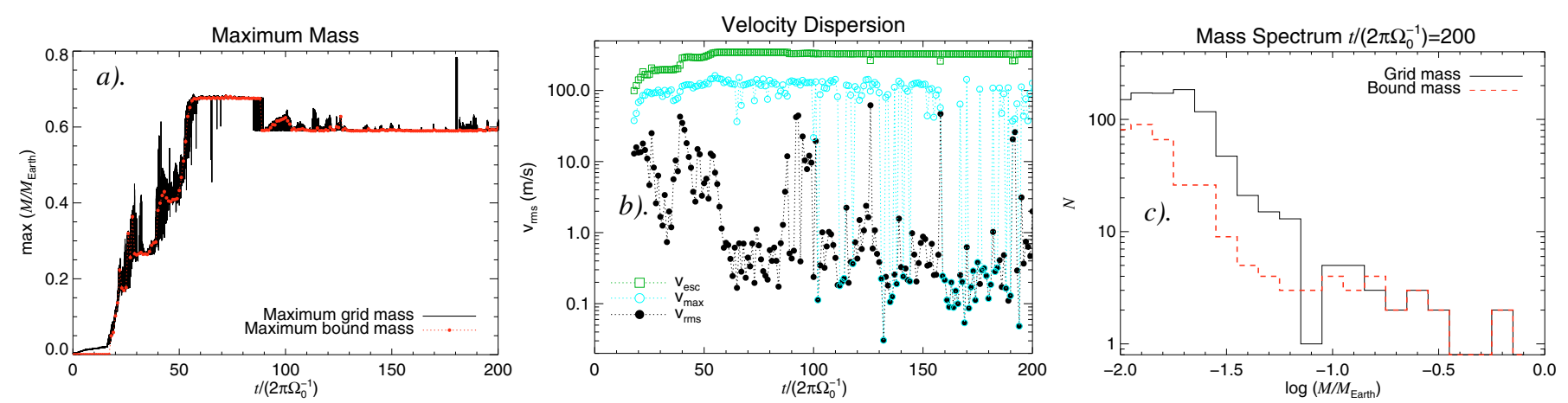

Fig. 2. a) Time evolution of the maximum mass of solids. The black solid line is the maximum mass of solids inside a single cell, and the red dotdashed line marks the mass that is gravitationally bound. The maximum mass settles at $0.59 M_{\oplus}$ from 90 orbits onwards; b) the internal velocity dispersion $v_{\text {rms }}$ of the most massive embryo, compared with its escaped velocity $v_{\text {esc }}$. The embryo is so firmly bound that even the maximum internal speed $v_{\max }$ is lower than the escape velocity. Throughout most of the simulation, the velocity dispersion is below $1 \mathrm{~m} \mathrm{~s}{ }^{-1}$; c) the mass spectrum by the end of the simulation. The line and color style is the same as in Fig. 2a. Twenty gravitationally bound embryos in the mass range $0.1-0.6 M_{\oplus}$ were formed in the vortices launched at the edges of the dead zone.

maximum mass that is gravitationally bound. We decide whether boundness is present based on two criteria. First, we consider the mass inside the Hill's sphere of the clump defined by the black line. Particles inside/outside the Hill's sphere are added/removed from the total mass, and the Hill's radius recomputed. The process is iterated until convergence. This positional criterion is followed by a dynamical one. We calculate the velocity dispersion $v_{\text {rms }}$ of the particles inside the Hill's radius, and compare its value with the escape velocity of the enclosed mass. If $v_{\text {rms }}<v_{\text {esc }}$, we consider that the cluster of particles is bound. We plot the velocity dispersion and escape velocity of the most massive clump in Fig. 2b. The first bound clumps appeared at 18 orbits, with masses of 0.050 and $0.036 M_{\oplus}$. At 20 orbits, four clumps of $0.1 M_{\oplus}$ are present. The mass is that of Mars, constituting a protoplanetary embryo. The efficiency of the vortex trapping mechanism can be more clearly appreciated if we consider the time elapsed between the rise of the Rossby vortices and the collapse of the trapped particles into a Mars sized object: only 5 orbits.

Two orbits later, the maximum mass increased to $0.22 M_{\oplus}$. Nine other clumps collapsed into embryos as well, five of them of mass above that of Mars. The maximum bound mass reached $0.67 M_{\oplus}$, but settled at $0.59 M_{\oplus}$ from 89 orbits until the end of the simulation at 200 orbits. We observe evidence that the mass loss episodes are due to tides from the gas, since the vortices concentrate sufficient gas to provide a considerable gravitational pull. In addition to tides, erosion (Cuzzi et al. 2008) also plays a role in disrupting clumps of smaller particles. We discuss this point further in the online supplement.

Figure $2 \mathrm{~b}$ also indicates that the velocity dispersion remained below $1 \mathrm{~m} \mathrm{~s}^{-1}$ for most of the simulation. This is of extreme importance because it implies that particle encounters are gentle enough for destructive collisions to be avoided. The opposite was reported for the massive disk models of Rice et al. (2006), where particle encounters in the spiral arms occurred at velocities comparable with the sound speed. In Fig. 2b, we plot the maximum speed $v_{\max }$ for comparison. It is evident that even $v_{\max }$ remains lower than the escape velocity. This indicates that even if destructive collisions occur, the fragments will remain bound, although the strong drag force felt by the fragments might delay any gravitational collapse.

We plot the mass spectrum of the formed embryos in Fig. 2c. The solid black line represents the mass of solids inside a cell of the simulation box, without information on boundness. We overplot the distribution of bound clumps with the red dashed line.
Over 300 bound embryos are formed by the end of the simulation, twenty of these being more massive than Mars. The mass spectrum follows a power law of index $-2.3 \pm 0.2$ in the interval $-2.0<\log \left(M / M_{\oplus}\right)<-1.2$.

The two most massive embryos have 0.59 and $0.57 M_{\oplus}$, and are located in the inner and outer edge, respectively.

A common trait of these embryos is that they consist of an overwhelming majority of particles of a single species. For instance, over $98 \%$ of the most massive embryo consists of $30 \mathrm{~cm}$ particles. Almost $97 \%$ of the second most massive one were $10 \mathrm{~cm}$ particles. In one embryo in the Mars mass bin, $94 \%$ of the particles were of $1 \mathrm{~cm}$ radius. These different particle radii reflect the variations in gas drag strengths experienced by particles of different size. Since particles of a given size drift in a similar way, their spatial convergence becomes easier than the convergence of particles of different sizes. The almost single-phasing of the size spectrum of the bound clumps also contributes to their low velocity dispersions. Since particles of different sizes experience different gas drags, their relative velocities are often far higher than the relative velocities between particles of identical size (Thébault et al. 2008). We indeed see that clumps with a more non-uniform distribution of particle sizes usually show larger velocity dispersions $\left(\approx 10\right.$ instead of $\left.\approx 1 \mathrm{~m} \mathrm{~s}^{-1}\right)$. It is an observed fact that planetesimals are formed of similarly sized particles (Scott \& Krot 2005). Although the constituent particles appear to be sub-mm grains, different nebula parameters could enable smaller grains to be trapped.

At higher resolution $\left(512^{2}\right.$, and $4 \times 10^{5}$ particles $)$, more bound clumps are formed, which extend to lower masses. More clumps containing similar quantities of particles of different sizes are observed, although the majority of clumps have nearly single phasing. The situation does not alter significantly when the number of particles is increased to $10^{6}$. In Fig. 3, we follow the collapse of one of these clumps at higher resolution. Although particles of different size are present inside the Hill's sphere during the first stages of collapse, most are expelled and the collapsed embryo consists primarily of $30 \mathrm{~cm}$ particles. A control run without self-gravity achieves higher cell concentration, due to the absence of gas tides. At later times, the clumps also tend to have low $\mathrm{rms}$ speeds and small $\mathrm{rms}$ radii $\left(\approx 10^{4} \mathrm{~km}\right)$, due to the efficient dynamical cooling provided by the drag force. Size segregation due to aerodynamical sorting is also observed in the control run. Inaba \& Barge (2006) reported destruction of the vortices by the drag force backreaction. We see a different 


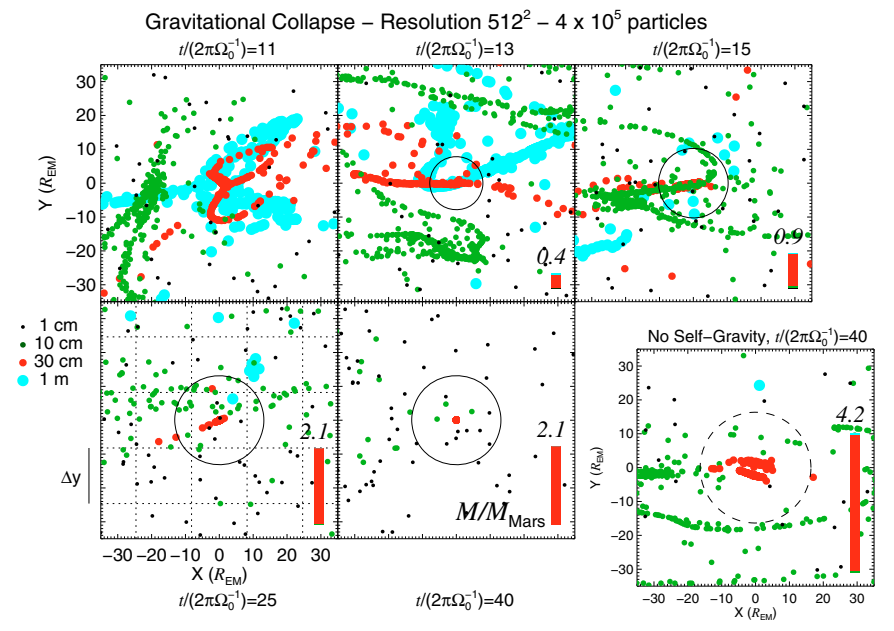

Fig. 3. Gravitational collapse of the particles into an embryo twice the mass of Mars. The unit of length is the Earth-Moon mean separation $\left(R_{\mathrm{EM}}\right)$. The lower left panel has the grid scale overplotted. The circle marks the Hill's radius. A control run without selfgravity attains higher cell concentration due to the absence of gas tides. The bar shows the mass of the embryo in units of $M_{\text {Mars }}$ and the fractional mass of each particle species.

effect, in which the particles alter the evolution of the Rossby vortices and generate a vorticity of their own.

\section{Conclusions}

In this Letter, we have shown that when modelling the selfgravity of the gas and solids in protoplanetary disks, gravitational collapse of the solids into Mars sized protoplanetary embryos occurs rapidly at the borders of the dead zone, where particles concentrate. We have also found that tides from the dense gaseous vortices may hinder the formation process significantly.

Studies considering the origin of oligarchs usually begin from a collection of 10-20 Mars sized objects (e.g. Kobuko et al. 2006). This Letter presents the first simulation in which a similar number of Mars-sized embryos are formed from centimeter and meter sized sized building blocks.

It is crucial to the model that particles grow to sufficient size, otherwise the drag force from the gas becomes too strong to allow any concentration. Testi et al. (2003) observed grains of up to $\mathrm{cm}$ sizes in the disk surrounding the pre-main-sequence star CQ Tauri, which provides some observational evidence that a sufficient number of particles of the required size may exist in true protoplanetary disks.
We emphasize again that the model used in this Letter is simplistic, and the conditions may differ with a more realistic treatment of the dead zone. Nevertheless, the mechanism presented in this Letter (as proposed originally by Varnière \& Tagger 2006) appears robust. It only requires the RWI to be excited in the borders between the active and dead zones, which in turn relies only on the slowdown of the accretion flow at this same border. Future studies should include a coagulation/fragmentation model such as those of Brauer et al. (2008) or Johansen et al. (2008), and focus on the precise state of flow at this transition region in global simulations, to address the question of how the RWI interacts with the MRI and non-ideal MHD in three dimensions.

Acknowledgements. Simulations were performed at the PIA cluster of the MaxPlanck-Institut für Astronomie and on the Uppsala Multidisciplinary Center for Advanced Computational Science (UPPMAX). We thank the referee, Dr. Laure Fouchet, for many helpful suggestions for improvement of the paper.

\section{References}

Baines, M. J., Williams, I. P., \& Asebiomo, A. S. 1965, MNRAS, 130, 63 Balbus, S. A., \& Hawley, J. F. 1991, ApJ, 376, 214

Barge, P., \& Sommeria, J. 1995, A\&A, 295, 1

Benz, W. 2000, SSRv, 92, 279

Brauer, F., Henning, Th., \& Dullemond, C. P. 2008, A\&A, 487, 1

Cuzzi, J. N., Hogan, R. C., \& Shariff, K. 2008 [arXiv: 0804 . 3526]

Gammie, C. F. 1996, ApJ, 457, 355

Haghighipour, N., \& Boss, A. P. 2003, ApJ, 583, 996

Inaba, S., \& Barge, P. 2006, ApJ, 649, 415

Johansen, A., Oishi, J. S., Mac Low, M.-M., Klahr, H., Henning, Th., \& Youdin, A. 2007, Nature, 448, 1022

Johansen, A., Brauer, F., Dullemond, C., Klahr, H., \& Henning, T. 2008, A\&A, 486, 597

Klahr, H., \& Bodenheimer, P. 2006, ApJ, 639, 432

Kokubo, E., Kominami, J., \& Ida, S. 2006, ApJ, 642, 1131

Kretke, K. A., \& Lin, D. N. C. 2007, ApJ, 664, 55

Kretke, K. A., Lin, D. N. C., Garaud, P., \& Turner, N. J. 2008 [arXiv: 0806.1521]

Kwok, S. 1975, ApJ, 198, 583

Li, H., Colgate, S. A., Wendroff, B., \& Liska, R. 2001, ApJ, 551, 874

Lyra, W., Johansen, A., Klahr, H., \& Piskunov, N. 2008, A\&A, 479, 883

Natta, A., Testi, L., Calvet, N., et al. 2007, in Protostars and Planets V, ed. B. Reipurth, D. Jewitt, \& K. Keil, 767

Paardekooper, S.-J. 2007, A\&A, 462, 355

Rice, W. K. M., Lodato, G., Pringle, J. E., Armitage, P. J., \& Bonnell, I. A. 2006, MNRAS, 372, 9

Scott, E. R. D., \& Krot A. N. 2005, in ASP Conf. Ser., 341, 15

Shakura, N. I., \& Sunyaev, R. A. 1973, A\&A, 24, 37

Testi, L., Natta, A., Shepherd, D. S., \& Wilner, D. J. 2003, A\&A, 403, 323

Thébault, P., Marzari, F., \& Scholl, H. 2008 [arXiv : 0806. 0819]

Varnière, P., \& Tagger, M. 2006, A\&A, 446, L13

Weidenschilling, S. J. 1977, MNRAS, 180, 5

Woitke, P., \& Helling, C. 2003, A\&A, 399, 297 


\section{Appendix A: Dynamical equations}

As stated in the main paper, we work in the thin disk approximation, using the vertically integrated equations of hydrodynamics

$$
\begin{aligned}
\frac{\partial \Sigma_{g}}{\partial t}= & -(\boldsymbol{u} \cdot \boldsymbol{\nabla}) \Sigma_{g}-\Sigma_{g} \boldsymbol{\nabla} \cdot \boldsymbol{u}+f_{\mathrm{D}}\left(\Sigma_{g}\right) \\
\frac{\partial \boldsymbol{u}}{\partial t}= & -(\boldsymbol{u} \cdot \boldsymbol{\nabla}) \boldsymbol{u}-\frac{1}{\Sigma_{g}} \boldsymbol{\nabla} P-\boldsymbol{\nabla} \Phi-\frac{\Sigma_{p}}{\Sigma_{g}} \boldsymbol{f}_{\mathrm{d}} \\
& +2 \Sigma_{g}^{-1} \boldsymbol{\nabla} \cdot\left(v \Sigma_{g} \boldsymbol{S}\right)+f_{v}\left(\boldsymbol{u}, \Sigma_{g}\right) \\
\frac{\mathrm{d} \boldsymbol{x}_{p}}{\mathrm{~d} t}= & \boldsymbol{v}_{p} \\
\frac{\mathrm{d} \boldsymbol{v}_{p}}{\mathrm{~d} t}= & -\boldsymbol{\nabla} \Phi+\boldsymbol{f}_{\mathrm{d}} \\
\Phi= & \Phi_{\mathrm{sg}}-\frac{G M_{\odot}}{r} \\
\nabla^{2} \Phi_{\mathrm{sg}}= & 4 \pi G\left(\Sigma_{g}+\Sigma_{p}\right) \delta(z) \\
P= & \Sigma_{g} c_{\mathrm{s}}^{2} \\
f_{\mathrm{d}}= & -\left(\frac{3 \rho_{g} C_{\mathrm{D}}|\Delta \boldsymbol{v}|}{8 a_{\bullet} \rho_{\bullet}}\right) \Delta \boldsymbol{v} .
\end{aligned}
$$

In the above equations, $G$ is the gravitational constant, $\Sigma_{g}$ and $\Sigma_{p}$ are the vertically integrated gas density and bulk density of solids, respectively, $\boldsymbol{u}$ represents the velocity of the gas parcels, $\boldsymbol{x}_{p}$ is the position and $\boldsymbol{v}_{p}$ is the velocity of the solid particles, $P$ is the vertically integrated pressure, $c_{\mathrm{s}}$ is the sound speed, $\Phi$ is the gravitational potential, $v$ is the viscosity, and $\mathbf{S}$ is the rate of strain tensor. The functions $f_{\mathrm{D}}\left(\Sigma_{g}\right)$ and $f_{v}\left(\boldsymbol{u}, \Sigma_{g}\right)$ are sixth order hyperdiffusion and hyperviscosity terms that provide extra dissipation close to the grid scale, explained in Lyra et al. (2008). They are required because the high order scheme of the Pencil code has too little overall numerical dissipation.

The function $\boldsymbol{f}_{\mathrm{d}}$ is the drag force by which gas and solids interact. In Eq. (A.8), $\rho_{\bullet}$ is the internal density of a solid particle, $a_{\bullet}$ its radius, and $\Delta \boldsymbol{v}=\boldsymbol{v}_{p}-\boldsymbol{u}$ its velocity relative to the gas. $C_{\mathrm{D}}$ is a dimensionless coefficient that defines the strength of the drag force.

\section{Appendix B: Drag force}

The drag regimes are controlled by the mean free path $\lambda$ of the gas, which can be expressed in terms of the Knudsen number of the flow past the particle $\mathrm{Kn}=\lambda / 2 a_{\bullet}$. High Knudsen numbers correspond to free molecular flow, or Epstein regime. Stokes drag is applicable to low Knudsen numbers. We use the formula of Woitke \& Helling (2003; see also Paardekooper 2007), which interpolates between Epstein and Stokes regimes

$C_{\mathrm{D}}=\frac{9 \mathrm{Kn}^{2} C_{\mathrm{D}}^{\mathrm{Eps}}+C_{\mathrm{D}}^{\mathrm{Stk}}}{(3 \mathrm{Kn}+1)^{2}}$.

where $C_{\mathrm{D}}^{\mathrm{Eps}}$ and $C_{\mathrm{D}}^{\mathrm{Stk}}$ are the coefficients of Epstein and Stokes drag, respectively. These coefficients are

$$
\begin{aligned}
& C_{\mathrm{D}}^{\mathrm{Eps}} \approx 2\left(1+\frac{128}{9 \pi \mathrm{Ma}^{2}}\right)^{1 / 2} \\
& C_{\mathrm{D}}^{\mathrm{Stk}}= \begin{cases}24 \mathrm{Re}^{-1}+3.6 \mathrm{Re}^{-0.313} & ; \mathrm{Re} \leq 500 \\
9.5 \times 10^{-5} \mathrm{Re}^{1.397} & ; 500<\mathrm{Re} \leq 1500 \\
2.61 & ; \operatorname{Re}>1500\end{cases}
\end{aligned}
$$

where $\mathrm{Ma}=|\Delta v| / c_{\mathrm{S}}$ is the Mach number, Re $=2 a_{\bullet} \rho|\Delta v| / \mu$ is the Reynolds number of the flow past the particle, and $\mu=$ $\sqrt{8 / \pi} \rho c_{\mathrm{s}} \lambda / 3$ is the kinematic viscosity of the gas.

The approximation for Epstein drag (Kwok 1975) connects regimes of low and high Mach number with a good degree of accuracy, and is more numerically friendly than the general case (Baines et al. 1965). The piecewise function for the Stokes regime are empirical corrections to Stokes law $\left(C_{\mathrm{D}}=24 \mathrm{Re}^{-1}\right)$, which only applies for low Reynolds numbers.

\section{Appendix C: Gas tides and mass loss}

The most remarkable feature of Fig. 3a of the main paper is the mass loss event at 90 orbits. It consists of the detachment of a $0.8 M_{\text {Mars }}$ object from the original cluster, of 6.7 $M_{\text {Mars }}$.

We observe evidence that this puzzling behaviour is due to gravitational tides from the gas. The gas is too pressuresupported to undergo collapse, but the vortices concentrate a sufficient amount of material to exert a considerable gravitational pull. We illustrate this in Fig. C.1, where we show the state of the disk before (at 80 orbits, Figs. C.1a-C.1d) the mass-loss episode and after (at 100 orbits, Figs. C.1e-C.1h). The plots show the surface densities of gas and solids, and the potential associated with them. Even though the clumping of solids yield a considerable gravitational pull (Figs. C.1d and C.1h), these figures indicate that the dominant contribution to the gravitational potential comes from the gas - more specifically from the vortices, where the gas density peaks at a value one order of magnitude higher than that of the initial conditions.

The most massive clump is located in the inner disk at $(X, Y)=(-0.40,-0.53)$ in Fig. C.1b, not clearly identifiable amidst the other particles trapped inside the vortex. However, the embryo is immediately observable as the bright point at $(X, Y)=(-0.65,-0.19)$ in Fig. C.1h (also visible in Fig. C.1f, albeit less prominently). There are two features in this plot that are worth noting. First, by comparing the location of the embryo in these figures with the location of the vortices, we notice that the planet has left its parental vortex. Second, the inner vortices have undergone the transition from the $m=3$ to the $m=2$ mode. Due to merging, their gas density has increased, with dramatic consequences for the embryos within them.

We assess how the gravity of the gas influences the motion of the particles in Figs. C.1i-C.11. In Fig. C.1i, we take a horizontal density slice at the position of the most massive embryo at 80 orbits. Figure C.1j is an enlargement of Fig. C.1i about $X=-0.53$, where the embryo is located. We see that the densities of solids and gas peak at similar values. The subsequent figures show the gravitational potential (Fig. C.1k) and acceleration (Fig. C.11) about the embryo. The gas produces a deeper gravitational well, albeit smoother than the one displayed by the solids. In the acceleration plot, it is seen that the pull of the gas is more significant than the pull of the embryo at a distance of only $0.26 \mathrm{AU}$ (0.03 in code units, corresponding to two grid cells) away from the center. And even where the pull of the solids is strongest (one grid cell away from the center), the gravity of the gas is still an appreciable fraction of the gravity of the solids. Tides from the gas are unavoidable.

It is beyond the scope of this paper to consider the full mathematical details of the theory of tides, especially because the two bodies (the vortex and the embryo) are extended. Instead, we consider the following toy model. The tidal force $F_{\mathrm{T}}$ experienced by the planet is proportional to the gradient of the acceleration $a$ induced by the gas. It is also proportional to the radius $R$ of the planet: $F_{\mathrm{T}} \propto R \boldsymbol{\nabla} a$. Since $\boldsymbol{\nabla} a=-\nabla^{2} \Phi$, according to the Poisson 

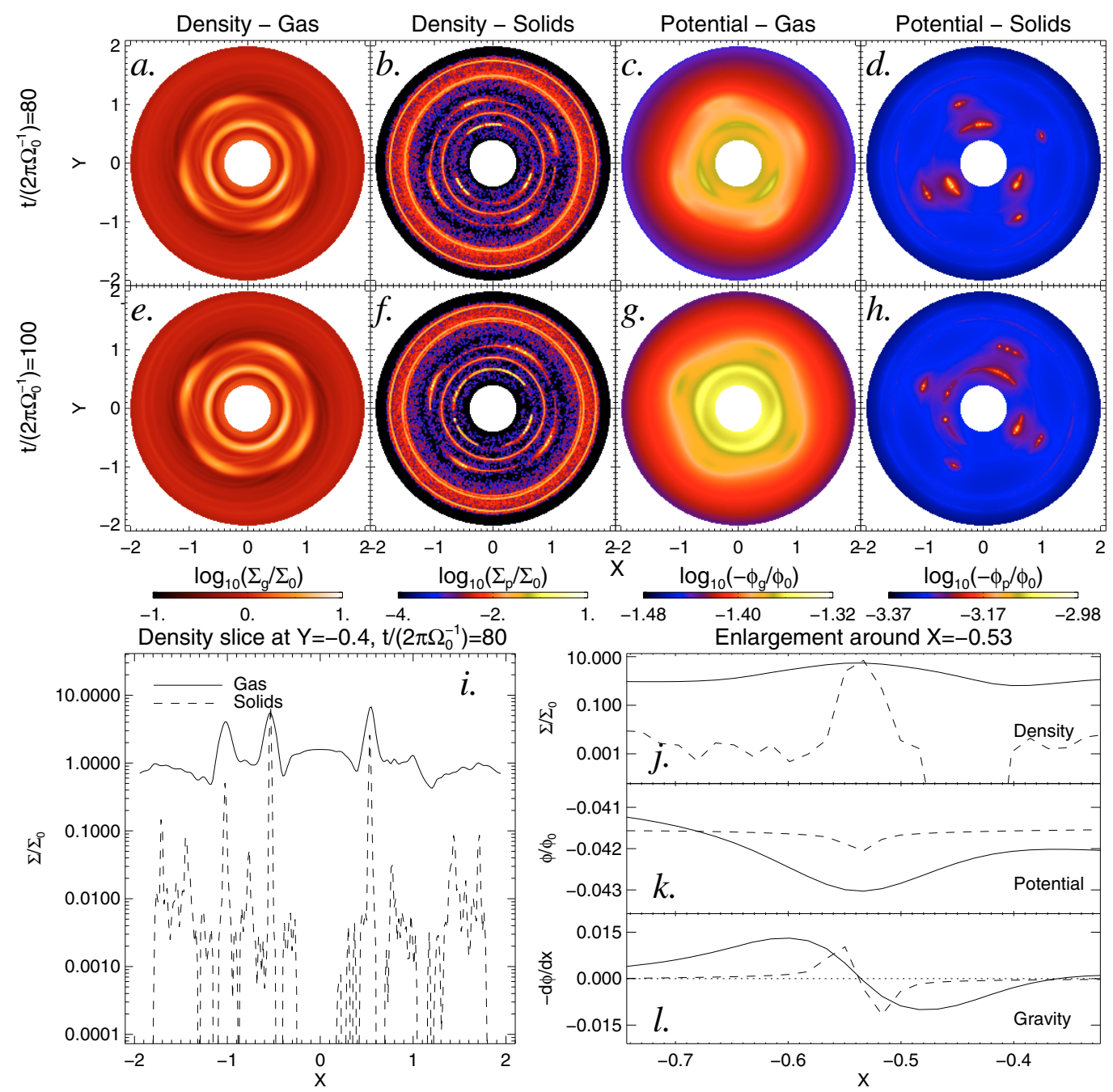

Fig. C.1. The state of the disk before (a)-d)) and after (e)-h)) the mass-loss episode. The conspicuous differences between them are due to the inner vortices passing from the $m=3$ to the $m=2$ mode. As a result, the the most massive embryo has left its parental vortex between one snapshot and another. It is seen as a bright spot in panels $f$ and $h$, at $(X, Y)=(-0.65,-0.19)$. In panel $b$ (before the mass loss), the embryo is at $(X, Y)=(-0.40,-0.53)$ but not easily identified among the swarm of solids inside the vortex. Panel $i$ shows a horizontal slice through this location, in which we see that the peak in the density of solids is not much higher than the gas density at the location of the embryo (panel $j$ ). Significant gas tides are expected because the gravitational potential (panel $k$ ) and acceleration (panel $l$ ) have similar contributions from gas and solid components.

equation, the tidal force is proportional to the local value of the density

$F_{\mathrm{T}} \propto R \rho_{g}$.

We consider the 3D volume density to avoid the requirement of using the Dirac delta in the 2D case. Considering the planet spherical, Newton's second theorem holds and we can assume that $F_{G}=-G M / R^{2}$ for the planet's (self-)gravitational force at its surface. Substituting $M=4 / 3 \pi \rho_{p} R^{3}$, we have $F_{G} \propto R \rho_{p}$, so

$\zeta=\frac{F_{\mathrm{T}}}{F_{G}} \propto \frac{\rho_{g}}{\rho_{p}}$,

i.e., the ratio of the disrupting tidal stresses to the self-gravitating forces that attempt to keep the planet together is directly proportional to the gas-to-solids ratio. At 80 orbits, as seen in Fig. C.1j, this ratio is around unity. As the vortices undergo the transition from the $m=3$ to the $m=2$ mode, their peak density increases (while the planet remains at constant mass). The tides eventually become sufficiently strong for a mass-loss event to occur.
We also witness some of the other, less massive, embryos being completely obliterated by the gas tides. Erosion is also important, since we observe a size dependency in this effect, with embryos consisting of $a_{\bullet}=10 \mathrm{~cm}$ particles being more prone to disruption than those consisting of $a_{\bullet}=30 \mathrm{~cm}$ particles. We performed tests that indicated that the erosion of bound clumps by ram pressure seen by Cuzzi et al. (2008) only occurs for clumps consisting of particles smaller than $\mathrm{cm}$-size for our initial nebula parameters, larger particles being more stable. However, when the gas density of the vortices reaches a maximum value a factor ten higher than the initial density, the gas drag also strengthens, shifting the onset of erosion towards larger particle radii.

The effect of tides will probably be less dramatic in 3D simulations because, as the particles settle into the midplane, the ratio of the volume gas density to the bulk density of solids $\rho_{g} / \rho_{p}$ is expected to be much lower than the ratio of the column gas density to the vertically integrated surface density of solids $\Sigma_{g} / \Sigma_{p}$. 